\title{
Study on Life Quality of Diabetes Patients and its Influencing
}

\section{Factors}

\author{
Gao Yan ${ }^{1}$, Zhou Dan $^{2}$, Bao Wuren ${ }^{1}$ \\ ${ }^{1}$ College of nursing, Inner Mongolia University for the Nationalities, Tongliao 028000, China \\ ${ }^{2}$ Affiliated hospital, Inner Mongolia University for the Nationalities, Tongliao 028000, China
}

\begin{abstract}
Objective: To investigate life quality of type II diabetes and its influencing factors of patients in Inner Mongolia area. Method: To compare life quality of patients with diabetes from community, together 161 cases taken by Affiliated Hospital of Inner Mongolia University for Nationalities' endocrine therapy area, with life quality of 150 cases of healthy people in the control group, so as to explore its influencing factors from June, 2014 to March, 2015. The general survey includes age, gender, severity of illness, education level and economic status, etc.; life quality of people can be scored by using Table SF-36, the investigation includes social function, mental health, body pain, emotional function and physiological function. Result: Score on life quality in social function, mental health, body pain and physiological function, scores in the study group are (59.6520.10), (53.5012.78), (70.0213.77), (74.1514.11) respectively, which is significantly lower than that in the control group, the difference is statistically significant $(p<0.05)$; the main factors of influencing life quality of patients in community diabetes including age, gender, education level, course of disease and degree of understanding disease. Conclusion: Life quality of diabetes patients is obviously lower than health group, and they need some effective interventions measures on residential status, age, educational level, course of disease, blood sugar control level, and complications and other issues to improve their life qualities.
\end{abstract}

Keywords: Type II Diabetes, Influencing Factors, Inner Mongolia Area

\section{Introduction}

Diabetes is caused by different etiology of metabolic disorders, symptoms of patients in I period mainly include clinical manifestations of chronic hyperglycemia, complicated with disorder or symptoms of insulin deficiency, which can cause metabolic disorder of carbohydrate, protein and fat, thus a variety of organs are easily got chronic injuries. Patients with severe renal failure, which can have severe threat to patient's health and life safety. With the continuous development of medical cause and health services as well as the continuous improvement of people's living standard, more and more attention has been paid to life quality of patients with diabetes. In this study, from June 2014 to March 2015, life quality of 161 patients with diabetes taken by Affiliated Hospital of Inner Mongolia University for Nationalities' endocrine therapy area and life quality of 150 cases of healthy people is compared and studied.

\section{Data and Method}

General data: June 2014 to March 2015, 161 patients with diabetes in community taken by Affiliated Hospital of Inner Mongolia University and150 cases of healthy people can be acted as study group. The average age is $(49.6 \pm 8.5)$ years old. The patients are diagnosed by clinical 
examination, so as to meet the diagnostic criteria of diabetes of patients in community, excluding patients with mental illness, communication disorder, malignant diseases, severe infection and severe organic disease, as well as patients, who are not cooperative with the investigation. Meanwhile, taking the same period of 150 cases of healthy people as the control group, the average age is (49.9 \pm 7.8$)$ years old. There is no significant difference in age, gender and other basic data between these two groups of people, which is comparable.

Method: patients are compared through questionnaire, as for people with lower education level can complete the questionnaire by asking them face to face. The content of the survey mainly includes two aspects, one is the general investigation, the other is survey on life quality. The general survey can be including age, gender, severity, education level, economic status and so on; by using Table SF-36 to score on life quality of people, the investigation can include social function, mental health, body pain, emotional function and physiological function, etc.. By comparing life quality of two groups of people, the influencing factors of life quality of patients with diabetes in community can be discussed.

Statistical method: In addition to data, SPSS18.0 statistical software is used. Normal measurement data can use " $\bar{x} \pm s "$ to present, the comparison between groups can use $t$ to test, comparison between groups of counting data can adopt test. When $\mathrm{P}<0.05$, the difference has statistical significance.

\section{Result}

Scores on social function, mental health, body pain, emotional function, physiological function and other life qualities of patients in the study group are significantly lower than that in control group $(\mathrm{P}<0.05)$, see Table 1 ; the main factors that can affect life quality of patients with diabetes in community including age, gender, education level, the course of disease and the understanding degree to the disease, see Table 2; factors that can have psychological effect on diabetic patients are age of patients and knowledge of understanding the disease; factors that can cause physical pain are mainly age of patients and course of disease; factors that can have effect on social function of patients are mainly the course of disease and education level; factor that can have effect on emotional function of patient is age, See table 3.

Table 1 Comparison of Scores on Life Quality in Two Groups

\begin{tabular}{ccccccc}
\hline Groups & $\begin{array}{c}\text { The number } \\
\text { of cases }\end{array}$ & $\begin{array}{c}\text { Social } \\
\text { function }\end{array}$ & Mental health & Somatic pain & $\begin{array}{c}\text { Emotional } \\
\text { function }\end{array}$ & Physiological function \\
\hline $\begin{array}{c}\text { Study } \\
\text { Group }\end{array}$ & 161 & $\begin{array}{c}59.65 \pm 20.1 \\
0^{\mathrm{a}}\end{array}$ & $53.50 \pm 12.78^{\mathrm{a}}$ & $\begin{array}{c}70.02 \pm 13.77 \\
\mathrm{a}\end{array}$ & $53.31 \pm 13.30^{\mathrm{a}}$ & $74.15 \pm 14.11^{\mathrm{a}}$ \\
$\begin{array}{c}\text { Control } \\
\text { group }\end{array}$ & 150 & $90.42 \pm 9.70$ & $88.31 \pm 12.75$ & $89.46 \pm 12.34$ & $87.34 \pm 14.14$ & $97.11 \pm 9.32$ \\
\hline
\end{tabular}


Table 2 Factors influence life quality of patients with diabetes in community

\begin{tabular}{cccccc}
\hline $\begin{array}{c}\text { Life quality/ } \\
\text { Influencing } \\
\text { factors }\end{array}$ & Age & Education level & $\begin{array}{c}\text { Course of } \\
\text { disease }\end{array}$ & Gender & $\begin{array}{c}\text { Disease } \\
\text { knowledge }\end{array}$ \\
\hline Social function & -0.362 & 0.342 & -0.369 & 0.006 & -0.286 \\
Mental health & -0.235 & 0.169 & -0.372 & 0.009 & 0.521 \\
Somatic pain & -0.364 & 0.267 & -0.308 & -0.299 & 0.265 \\
Emotional & -0.453 & 0.294 & -0.421 & 0.142 & -0.057 \\
function & & & & & 0.427 \\
Physiological & -0.542 & 0.312 & -0.378 & -0.062 & \\
function & & & & & \\
\hline
\end{tabular}

Table 3 Effect of Stepwise Regression Analysis on Life Quality of Patients with Diabetes

\begin{tabular}{cccc}
\hline Life quality & Influencing factors & Regression coefficient & The value of P \\
\hline \multirow{2}{*}{ Social function } & Course of disease & -0.302 & 0.013 \\
& education level & 0.256 & 0.024 \\
\multirow{2}{*}{ Mental health } & Disease knowledge & 0.453 & $<0.001$ \\
& Age & -0.306 & 0.006 \\
Somatic pain & Course of disease & -0.248 & 0.012 \\
& Age & -0.375 & 0.006 \\
Emotional function & Age & -0.467 & $<0.001$ \\
& Disease knowledge & 0.369 & 0.004 \\
\hline
\end{tabular}

\section{Discussion}

Nowadays, the health service cause in China has focused on the prevention and control of chronic diseases. Diabetes is one of the four major chronic diseases, the prevalence rate is being increased year by year. Once the blood glucose level of patients is in a high state for a long time, it will cause eye, heart, kidney, nerves and blood vessels and other functional disorders. In the past, the main reference to the patient's blood glucose level and glycosylated hemoglobin and other biochemical indicators to determine therapeutic targets, disease control and curative effect judgment. Due to the formation of modern medical model, the clinical objective of treating diabetes is not only in controlling blood glucose level, it is also including the prevention of complications, the relief of disease symptoms and the improvement of life quality. Life quality can play a very important role in evaluating patients' disease and treating mental health, physical function and social relations. Through the investigation of life quality of patients with diabetes in community, it can analyze the influencing factors and give targeted therapy, which can improve life quality of patients. There are many factors that can affect life quality of patients with diabetes in community.

\section{Conclusion}

The result of this study showed that scores of life quality in study group on social function, mental health, body pain, emotional function and physiological function was significantly lower than that in the control group $(\mathrm{P}<0.05)$; factors that can affect life quality of patients with diabetes in community including age, education level, the course of disease, gender and the degree of understanding and so on; factors that can have psychological effect on diabetic patients mainly including age of patients and knowledge of understanding the disease; factors that can cause physical 
pain including age of patients and course of disease; factors that can influence the social function of patients mainly including course of disease and education level; factors that can influence patient's emotional function mainly including age, which illustrated us that life quality of patients with diabetes in community was mainly related to age, education level, course of disease, gender and the understanding of the disease. People can help elderly patients with diabetes, so that disease can be early detection, early diagnosis and early treatment, preventing the occurrence of complications, relieving the clinical symptoms of patients, treating them with psychotherapy and giving health education guidance, which has significant meaning to improve life quality of patients with diabetes.

\section{Reference}

[1] Yang, W., Lu, J., Weng, J., Jia, W., Ji, L., Xiao, J., ... \& Zhu, D. (2010). Prevalence of diabetes among men and women in China. New England Journal of Medicine, 362(12), 1090-1101.

[2] Yong-hong, W. A. N. G. (2008). Health condition analysis of civil servants in Chongqing city [J]. Journal of Chongqing Medical University, 1, 030.

[3] Guichun, H., Liying, Z., \& Yajie, L. (2010). Research status quo of self-monitoring of diabetes mellitus patients. Chinese Nursing Research, 35, 008.

[4] WANG, Q., CAO, H. W., \& LU, F. (2012). The Research on Domestic Female Teachers' Participation in Physical Activity Characteristics in Primary and Middle School. Journal of Anshan Normal University, 2, 022.

[5] Han, C., Zhang, M., Luo, X., Wang, C., Yin, L., Pang, C., ... \& Li, L. (2016). Secular trend of prevalence of type 2 diabetes in adults in China from 1995 to 2014: A meta - analysis. Journal of Diabetes.

[6] Yuan, F., Qian, D., Huang, C., Tian, M., Xiang, Y., He, Z., \& Feng, Z. (2015). Analysis of awareness of health knowledge among rural residents in Western China. BMC public health, 15(1), 1. 